\title{
A PARAMETRIC TESTING OF THE FIREFLY ALGORITHM IN THE DETERMINATION OF THE OPTIMAL OSMOTIC DRYING PARAMETERS OF MUSHROOMS
}

\author{
Julian Scott Yeomans \\ OMIS Area, Schulich School of Business, York University \\ Toronto, ON, M3J 1P3 Canada
}

\begin{abstract}
The Firefly Algorithm (FA) is employed to determine the optimal parameter settings in a case study of the osmotic dehydration process of mushrooms. In the case, the functional form of the dehydration model is established through a response surface technique and the resulting mathematical programming is formulated as a non-linear goal programming model. For optimization purposes, a computationally efficient, FA-driven method is used and the resulting optimal process parameters are shown to be superior to those from previous approaches. The final section of this study provides a computational experimentation performed on the FA to analyze its relative sensitivity over a range of the two key parameters that most influence its running time.
\end{abstract}

\section{Introduction}

Commercial agronomy represents a multibillion dollar, worldwide enterprise. Within this industry, the annual global production of mushrooms currently exceeds 6 million tonnes [1]. As with many fresh fruits and vegetables, the high moisture content of mushrooms renders them highly perishable and, due to various enzymatic, microbial and chemical reactions, they commence deterioration immediately upon harvesting [2] [3]. Therefore, it becomes imperative to determine effective preservation methods that retain the overall quality and desirable features of the product. The preservation of many agricultural commodities has often been accomplished by employing various combinations of drying using heat processing and dehydration[2] [3] [4]. The dehydration of fresh produce generally extends their storage lives, decreases their shipping weights, and reduces the need for special packing requirements [2]. However, hot-air dried products conventionally processed using tray, vacuum, or cabinet dryer techniques have not received widespread acceptance due to the perceived diminished quality of the endproduct [2] [4] [5].

Recently, osmotic dehydration has been introduced as a practical alternative preservation approach that is capable of producing a higher quality final product [6]. In osmotic dehydration, fresh produce is immersed in a hypertonic solution where the water content from the cells of the produce is transferred into the solution due to the relative differences in their solute concentrations [6]. In this processing, osmotic dehydration removes a desired portion of the water from within the fresh produce resulting in a product of intermediate moisture content [7] [8]. Simultaneously, a corresponding transfer of solid materials (normally sugar and/or salt) occurs from the solution into the product [6] [9] [10]. In terms of final product quality relative to standard hot air drying methods, osmotic dehydra- 
tion causes only minimal thermal degradation due to the low temperatures involved [6] [11] [12] [13].

Osmotic dehydration of fresh agricultural commodities can also be used as a pre-treatment to supplemental dry-processing because it improves many sensory, functional and nutritional properties [14]. The quality of the subsequent product is superior to one without pre-treatment due to (i) the improvements to texture of the fruits and vegetables, (ii) the stability of the colour pigmentation during storage, and (iii) increases in the solid gain transfer of sugar and salt from the hypertonic solution [2] [11]. Thus, in conjunction with other ensuing drying technologies, osmotic dehydration produces a superior quality, shelf-stable product for both local consumption and export markets.

Water removal during the dehydration process is influenced by many factors such as type and concentration of osmotic agents, temperature, circulation/agitation of solution, solution-to-sample ratio, thickness of food material, and any pre-treatments [4] [6]. While an expanding market currently exists for osmo-convective dehydrated fruits and vegetables in both domestic and world markets, only limited efforts have been undertaken to optimize the requisite osmotic process parameters [2] [11] [15] [16] [17] [18]. Specifically, an effective analysis of the mass transport occurring within the osmosis process measured in terms of water loss and solid (sugar, salt) gains is of considerable commercial and practical relevance [4] [6] [10].

In this study, the functional form of the osmotic dehydration process for mushrooms is constructed using a standard response surface technique [17] [18] [19] [20] [21]. The format of the resulting optimization model is shown to be a non-linear goal programming problem [15] [16] [17] [18]. This study employs the Firefly Algorithm (FA) [17] [22] [23] [24] to determine the optimal osmotic parameters for the mushroom dehydration case considered in [2]. It can be shown that the resulting osmotic process parameters produced by the FA are superior to those from the previous approaches. Extending the approach introduced in [25], the final portion of the study provides an extensive computational experimentation performed on the FA using the osmotic dehydration model to determine the relative sensitivity of the procedure over ranges of the two key parameters that most influence its running time.

\section{Functional Form and Mathemat- ical Model of the Osmotic Dehy- dration Process}

The first section of the analysis examines the dehydration case of mushrooms taken from [2]. In the case, a brine solution is employed for dehydration and the solid gain corresponds to the transport of salt from the brine into the mushrooms. The first step requires the construction of an appropriate model of the responses to the three main osmotic process parameters - (i) solution temperature, (ii) hypertonic solution concentration and (iii) duration of osmosis - on the water loss and solid gain of the mushrooms. This functional representation can then be used to predict the water loss and salt gain impacts in the mushrooms over the requisite experimental ranges of the three designated parameters. Once the appropriate model has been constructed, the next step is to optimize this model in order to determine the maximum water loss and the optimum salt gain achieved during dehydration. In the subsequent formulations, let $T$ represent the brine solution temperature in ${ }^{\circ} \mathrm{C}, C$ be the salt solution concentration in percent, and $D$ be the duration of the osmosis measured in minutes. For the response variables, let $W L$ be the percentage of water loss and $S G$ represent the solid gain of the product during the dehydration process. In this instance, $S G$ corresponds to the percentage of salt gain in the mushrooms.

Response surface methods are statistical techniques frequently used for optimization in empirical studies [19] [20] [21]. Response surfaces employ quantitative data in appropriately designed experiments to simultaneously ascertain the various variable relationships within multivariate problems [21]. The equations constructed describe the effect of various test variables on responses, determine interrelationships among the test variables and represent the combined effect of all test variables in any response. Response surfaces enable an experimenter to undertake an efficient exploration of a process or system [20] [21]. These approaches have frequently been used in the optimization of food processes [2] [11] [26] [27] [28] [29] [30] and will, consequently, be employed in this study to determine the appropriate mathematical representation. The proposed model can then be used to predict the 
water loss and salt gain in the dehydration of mushrooms over the different experimental ranges for the process durations, brine concentrations and solution temperatures.

For the osmotic dehydration process, it should be noted that the exact mathematical representation for the relationship between the parameters remains unknown. Thus a response surface methodology enables an empirical approximation to it using efficient experimental design techniques [20] [21]. The specific testing design actually contains the three variables $(T, C, D)$ each set at three levels using the data taken from [2] in order to determine the corresponding water loss (WL) and salt gain $(S G)$ responses. The design for the various combinations of input variables and levels requires the various experimental combinations shown in Table 1 (see [2]), while the values determined for the response variables $W L$ and $S G$ appear in last two columns of Table 1.

Based upon the response surface experimental design appropriately applied to the water loss and the salt gain outputs of Table 1 [19] [20] [21], the functional equations empirically determined for responses are:

$$
\begin{gathered}
W L=19.58-0.13 T+1.7 C+0.98 D+0.00357 T D \\
+0.00673 C D-343 C^{2}-0.0106 D^{2} \\
S G=-13.87+0.11 T+1.09 C+0.14 D- \\
0.000973 T^{2}-0.0296 C^{2}-0.00129 D^{2} .
\end{gathered}
$$

Mehta et al. [2] established organoleptic ranges for the osmotic dehydration parameters and restricted their search for best parameter settings to values within these ranges. Organoleptic properties refer to sensory aspects of food including taste, sight, smell, touch, dryness, moisture content, and stalefresh factors. In order to find values for the osmotic dehydration parameters, Mehta et al. [2] constructed a number of contour plots by varying the values of the three variables and observed the effect that these had on their response functions. By superimposing these contours onto a single chart, the best settings for the temperature, concentration, and duration variables were determined to be $44.89^{\circ} \mathrm{C}$, $16.53 \%$ and 47.59 minutes, respectively. These settings generate responses of $40.55 \%$ for water loss and $2.98 \%$ for salt gain (see Table 2).

\section{A Goal Programming Formula- tion for Setting Osmotic Dehy- dration Parameters}

The determination of the parameters settings can be viewed as a multi-response optimization process and could, therefore, be transformed into a corresponding mathematical programming model [15] [16] [17] [18]. In this section, this formulation will be accomplished by converting the parameter setting process into an equivalent goal programming format.

Based upon the organoleptic requirements established for the parameters and response functions in [2], the technical constraints for the problem can be specified as:

$$
\begin{aligned}
26.18 & \leq W L \leq 45.04 \\
0.33 & \leq S G \leq 3.24 \\
35 & \leq T \leq 55 \\
10 & \leq C \leq 20 \\
30 & \leq D \leq 60
\end{aligned}
$$

Additional organoleptic preferences can be applied to the responses and variables for the solution. The targets for these desired criteria are summarized in Table 3. From a hierarchical preference attainment perspective, several of these criteria can be recognized as more important attributes to achieve than the others. Namely, from a dehydration perspective, the water loss should be as high as possible within the indicated range, while from a taste perspective, the salt gain needs to be as close to $2.98 \%$ as possible. The relative importance for the achievement of these hierarchy targets is indicated in the last column of Table 3.

Hence, from a mathematical perspective, each of these desired targets can be specified as a definitive goal and the entire formulation can then be transformed into a conventional goal programming problem. An objective function that appropriately penalizes deviations from the desired targets must be created and, in the subsequent mathematical programming formulation, a percentage deviation objective weighted by the relative importance of each goal is employed. Consequently, the problem of determining osmotic dehydration parameter values can be transformed into the following non-linear goal programming formulation. 
Table 1. Response Surface Experimental Design Layout for 3 Variables and 3 Levels

\begin{tabular}{|l|l|l|l|l|l|l|l|}
\hline $\begin{array}{l}\text { Level } \\
\text { for } \\
\boldsymbol{T}\end{array}$ & $\begin{array}{l}\text { Temperature } \\
\left({ }^{o} \mathbf{C}\right)\end{array}$ & $\begin{array}{l}\text { Level } \\
\text { for } \\
\boldsymbol{C}\end{array}$ & $\begin{array}{l}\text { Concentration } \\
(\boldsymbol{\%})\end{array}$ & $\begin{array}{l}\text { Level } \\
\text { for } \\
D\end{array}$ & $\begin{array}{l}\text { Duration } \\
(\text { Mins) }\end{array}$ & $\begin{array}{l}\text { Water } \\
\text { Loss }(\%)\end{array}$ & $\begin{array}{l}\text { Salt } \\
\text { Gain } \\
(\%)\end{array}$ \\
\hline 1 & 55 & 1 & 20 & 0 & 45 & 44.93 & 3.24 \\
\hline 1 & 55 & -1 & 10 & 0 & 45 & 36.38 & 1.03 \\
\hline-1 & 35 & 1 & 20 & 0 & 45 & 39.70 & 2.56 \\
\hline-1 & 35 & -1 & 10 & 0 & 45 & 29.92 & 0.59 \\
\hline 1 & 55 & 0 & 15 & 1 & 60 & 43.92 & 2.90 \\
\hline 1 & 55 & 0 & 15 & -1 & 30 & 34.23 & 2.24 \\
\hline-1 & 35 & 0 & 15 & 1 & 60 & 37.09 & 2.34 \\
\hline-1 & 35 & 0 & 15 & -1 & 30 & 29.54 & 1.73 \\
\hline 0 & 45 & 1 & 20 & 1 & 60 & 45.04 & 3.03 \\
\hline 0 & 45 & 1 & 20 & -1 & 30 & 35.51 & 2.22 \\
\hline 0 & 45 & -1 & 10 & 1 & 60 & 33.69 & 1.06 \\
\hline 0 & 45 & -1 & 10 & -1 & 30 & 26.18 & 0.33 \\
\hline 0 & 45 & 0 & 15 & 0 & 45 & 38.05 & 2.57 \\
\hline 0 & 45 & 0 & 15 & 0 & 45 & 38.44 & 2.64 \\
\hline 0 & 45 & 0 & 15 & 0 & 45 & 38.27 & 2.64 \\
\hline 0 & 45 & 0 & 15 & 0 & 45 & 38.55 & 2.79 \\
\hline 0 & 45 & 0 & 15 & 0 & 45 & 38.60 & 2.82 \\
\hline
\end{tabular}

Table 2. Best Osmotic Dehydration Parameters Determined by Mehta et al

\begin{tabular}{|c|c|c|c|c|}
\hline $\begin{array}{l}\text { Temperature } \\
\left({ }^{o} \mathbf{C}\right)\end{array}$ & $\begin{array}{l}\text { Concentration } \\
(\%)\end{array}$ & $\begin{array}{l}\text { Duration } \\
\text { (Mins) }\end{array}$ & $\begin{array}{l}\text { Water } \\
\text { Loss }(\%)\end{array}$ & $\begin{array}{ll}\text { Salt Gain } \\
(\%)\end{array}$ \\
\hline 44.89 & 16.53 & 47.59 & 40.55 & 2.98 \\
\hline
\end{tabular}

Table 3. Ranges for Process Variables and Response Goals in the Osmotic Dehydration

\begin{tabular}{|l|l|l|l|l|l|}
\hline Parameter & Goal & Requirement & $\begin{array}{l}\text { Lower } \\
\text { Limit }\end{array}$ & $\begin{array}{l}\text { Upper } \\
\text { Limit }\end{array}$ & Relative Importance \\
\hline $\begin{array}{l}\text { Temperature } \\
\left({ }^{\circ} \mathrm{C}\right)\end{array}$ & 1 & Minimize & 35 & 55 & Important \\
\hline $\begin{array}{l}\text { Concentration } \\
(\%)\end{array}$ & 2 & Minimize & 10 & 20 & Important \\
\hline Duration (Mins) & 3 & Minimize & 30 & 60 & Important \\
\hline Water Loss (\%) & 4 & Maximize & 23.02 & 44.05 & Very Important \\
\hline Salt Gain $(\%)$ & 5 & Target $=2.98$ & 0.33 & 3.24 & Very Important \\
\hline
\end{tabular}




$$
\begin{gathered}
\text { Minimize } W_{1} * P_{1}+W_{2} * P_{2}+W_{3} * P_{3}+W_{4} * N_{4}+ \\
W_{5} *\left(P_{5}+N_{5}\right) \\
\text { s.t. } P_{1}=T-35 \\
N_{1}=55-T \\
P_{2}=C-10 \\
N_{2}=20-C \\
P_{3}=D-30 \\
N_{3}=60-D \\
P_{4}=W L-26.18 \\
N_{4}=45.04-W L \\
P_{5}=S G-2.98 \\
N_{5}=2.98-S G \\
P_{6}=S G-0.33 \\
N_{6}=3.24-S G \\
P_{i} \geq 0, N_{i} \geq 0 \\
i=1,2,3,4,5,6
\end{gathered}
$$

In order to complete the transformation of the problem into the series of defined goals, several additional deviation variables have been introduced. Namely, for the goal model, define $P_{i}$ and $N_{i}, i=1$ to 6 , to be the positive and negative deviations, respectively, from the disparate goal targets and constraint limits shown for the variables in Table 3. Let $W_{i}$ correspond to weighting factors applied to goal $i, i=1$ to 5 , to reflect the relative importance in achieving that goal's target. Each $W_{i}$ also contains the appropriate denominator constant required to transform the deviation variables into the requisite percentage deviation value format. Thus, solving the goal programming model would be equivalent to determining optimal parameter values for the osmotic dehydration process.

\section{A Goal Programming, Firefly Algorithm-driven Optimization Approach}

While numerous different techniques could have been used to solve the resulting optimization problem, the method actually applied uses an FA procedure. For optimization, Yang [23] has proved that the FA is more computationally efficient than other such commonly-used metaheuristics as simu- lated annealing, enhanced particle swarm optimization, and genetic algorithms. Thus, the FA represents a very computationally efficient solution procedure. This section provides a brief outline of the FA procedure, while more comprehensive descriptions can be found in [22] and [23].

The FA is a population-based, nature-inspired metaheuristic in which each firefly within the population corresponds to one potential solution to the problem. All FA procedures employ three specific rules: (i) The fireflies within a population are unisex, so that one firefly will be attracted to other fireflies irrespective of their sex; (ii) Attractiveness between any two fireflies is proportional to their brightness, implying that the less bright firefly will move towards the brighter one; and (iii) The explicit brightness of any firefly is explicitly determined by the corresponding value of its objective function. For maximization problems, the brightness can be considered proportional to the value of the objective function. Yang (2010) demonstrates that the FA approaches the global optima whenever the number of fireflies $n \infty$ and the number of iterations $t$, is set so that $t>>1$. In reality, the FA has been shown to converge extremely quickly into both local and global optima [22] [23]. The basic operational steps of the FA are summarized in Table 4 [23].

There are two important requirements that must be determined for the FA: (i) the variation of light intensity and (ii) the formulation of attractiveness. Without loss of generality, it can always be assumed that the attractiveness of a firefly is determined by its brightness which in turn is associated with the encoded objective function. In the simplest case, the brightness of a firefly at a particular location $\boldsymbol{X}$ would be its calculated objective value $F(\boldsymbol{X})$. However, the attractiveness, $b$, between fireflies is relative and will vary with the distance $r_{i j}$ between firefly $i$ and firefly $j$. In addition, light intensity decreases with the distance from its source, and light is also absorbed in the media, so the attractiveness should be allowed to vary with the degree of absorption. Consequently, the overall attractiveness for a firefly specified as

$$
\beta=\beta_{0} \exp \left(-\gamma r^{2}\right)
$$

where $\beta_{0}$ is the attractiveness at distance $r=0$ and $\gamma$ is the fixed light absorption coefficient for the specific medium. The distance $r_{i j}$ between any two 
Table 4. Pseudo Code of the Firefly Algorithm

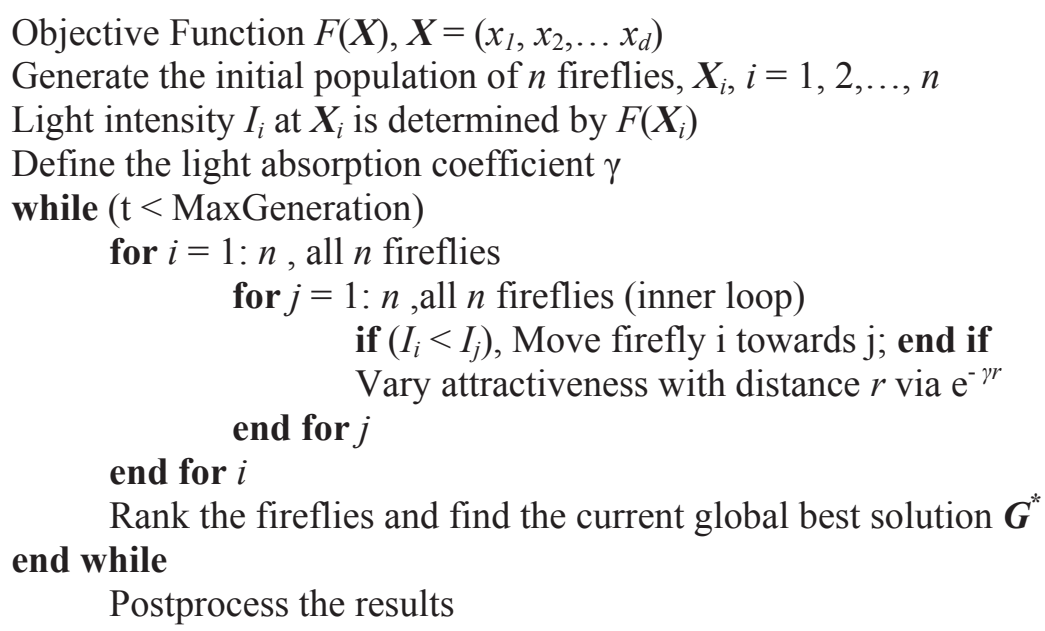

fireflies $i$ and $j$ located at $\boldsymbol{X}_{i}$ and $\boldsymbol{X}_{j}$, respectively, is calculated using the Euclidean norm. The movement of a firefly $i$ that is attracted to another more attractive (i.e. brighter) firefly $j$ can therefore be specified as

$$
X_{i}=X_{i}+\beta_{0} \exp \left(-\gamma\left(r_{i j}\right)^{2}\right)\left(X_{i}--X_{j}\right)+\alpha \varepsilon_{i} .
$$

In this expression, the second term is the movement due to relative attraction and the third term represents a randomization component. Yang [23] states that $\alpha$ represents a randomization parameter selected within the range $[0,1]$ and $\varepsilon_{i}$ corresponds to a vector of random numbers drawn from either a standard normal or a uniform (generally $[-0.5,0.5]$ ) distribution. This expression represents a random walk biased toward brighter fireflies. Furthermore, if $\beta_{0}=0$, it becomes a simple random walk. The parameter $\gamma$ portrays the variation of attractiveness and its value can determine the algorithm's rate of convergence. Normally, $\gamma$ is typically set between 0.1 to 10 in most practical applications [23]. For the computational approaches for the FA considered in this study, the variation of attractiveness parameter $\gamma$ is fixed at 5 while the randomization parameter $\alpha$ is initially set at 0.6 , but is then gradually decreased to a value of 0.1 as the procedure approaches its maximum number of iterations (see [23]).

Optimizing the goal programming problem using the FA-driven procedure, the best process parameters for the osmotic dehydration of the mushrooms were calculated. The resulting values are shown in Table 5. Comparing these values to those found by
Mehta et al. [2], it can be seen that the salt concentration increases by $2.5 \%$, the required temperature increases by $9^{\circ} \mathrm{C}$, while the duration of dehydration remains essentially unchanged. In terms of the two key response variables, the resulting water loss increases by $4.5 \%$, while the salt gain remains at its desired organoleptic target of $2.98 \%$. Consequently, since the water loss response - which is obviously the fundamental feature of the osmotic dehydration process - has been increased significantly from that determined in [2], this goal programming solution provides a significant improvement.

In any given optimization problem, for a very large number of fireflies $n>>k$ where $k$ is the number of local optima, the initial locations of the $n$ fireflies should be distributed as uniformly as possible to ensure that a comprehensive search throughout the search domain occurs. As the FA proceeds, the fireflies should converge into all of the local optima, including the global ones. By comparing the best solutions among all these optima, the global optima can easily be determined. As noted above, the FA approaches the global optima whenever the number of fireflies $n \rightarrow \infty$ and the number of iterations $t$, is set so that $t>>1$ [23]. In reality, the FA has a tendency to converge very quickly into both local and global optima [22] [23] [31].

As can be observed in Table 4, the two parameters that most directly impact the solution running time of the FA are the values selected for $n$ and $t$. Obviously, for practical applications, the desire is to be able to determine the best solution in the shortest period of time. This would correspond to setting $n$ 
Table 5. Optimal Process Parameters Determined for the Osmotic Dehydration of Mushrooms

\begin{tabular}{|l|l|l|l|l|l|}
\hline & $\begin{array}{l}\text { Temperature } \\
\left({ }^{\circ} \mathbf{C}\right)\end{array}$ & $\begin{array}{l}\text { Concentration } \\
(\boldsymbol{\%})\end{array}$ & $\begin{array}{l}\text { Duration } \\
(\text { Mins })\end{array}$ & $\begin{array}{l}\text { Water } \\
\text { Loss }(\%)\end{array}$ & $\begin{array}{l}\text { Salt } \\
\text { Gain } \\
(\boldsymbol{\%})\end{array}$ \\
\hline Mehta et al. [2] & 44.89 & 16.53 & 47.59 & 40.55 & 2.98 \\
\hline FA Solution & 54.043 & 19.031 & 46.777 & 45.04 & 2.98 \\
\hline
\end{tabular}

and $t$ at the minimum possible values that produce the best solution(s). Using terminology from computational complexity, the search time for the FA is linear in $t$, but is a second order polynomial in $n$.

However, since the FA's search process incorporates random components within its solution search, the parameter setting is clearly not a strictly deterministic issue - determining appropriate values for $n$ and $t$ reflects a component of choice on the part of the decision-maker. Consequently, an subsequent sensitivity analysis was performed for different combinations of the number of fireflies, $n$, and the number of iterations, $t$, on the solution quality. Specifically, the value of the firefly parameter was set at $n=20,50,100,150,200,250,250,500$ and the value for the number of iterations was set at $t$ $=100,250,500,1000,1500,2500$. For 30 runs of each parametric combination of fireflies and iterations, the corresponding responses for the water loss and salt gain were recorded. The average values of these responses over the 30 runs per combination are provided in Table 6 and visual representation of these values appears in Figures 1 and 2, respectively.

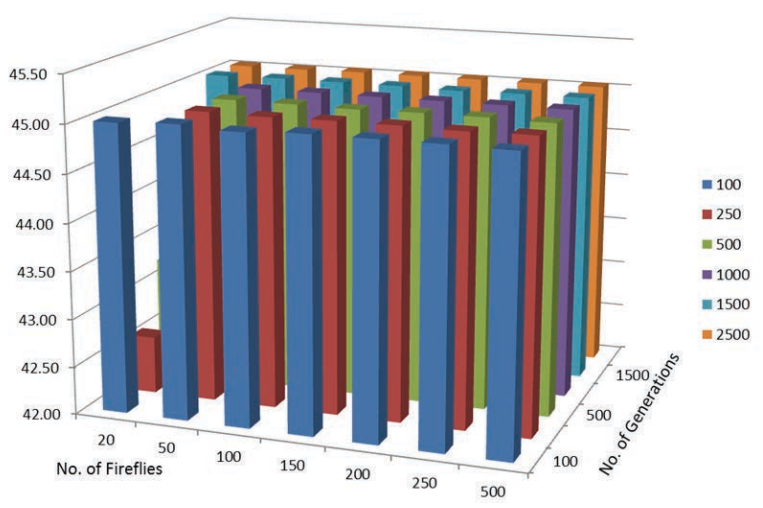

Figure 1. Average Water Loss (\%) in the Mushrooms for Different Parameter Settings of the Firefly Algorithm

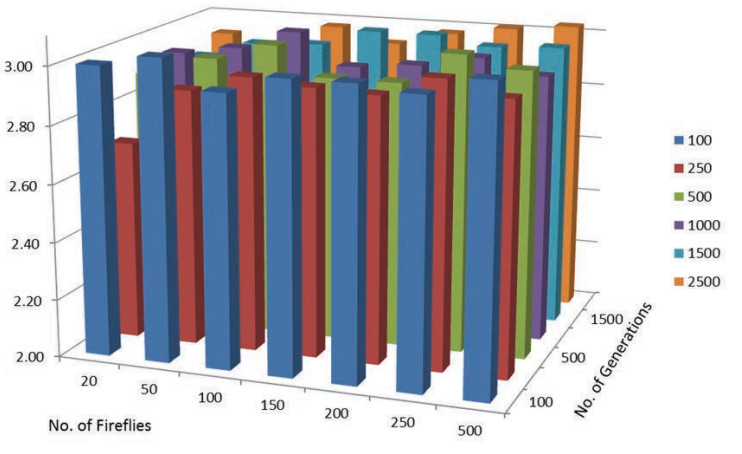

Figure 2. Average Salt Gain (\%) in the Mushrooms for Different Parameter Settings of the Firefly Algorithm

As might have been reasonable to anticipate $a$ priori, it is interesting to observe that more consistent solutions (i.e. where the average values are closer to optimal) are obtained when the values for either the number of fireflies or the number of iterations are relatively larger. Namely larger values of $n$ or $t$ tend to produce solutions closer to the actual optimal solution on average, while combinations involving smaller parameter values tend to exhibit more solution variability in terms of both water loss and salt gain. While there are multiple approaches that can be undertaken to parse these results, Figures 3 and 4 provide comparisons of the average water loss and salt gain responses obtained for the minimum and maximum number of iterations considered in the experimentation. From Figures 3 and 4 , it can be observed that at $t=2500$, the FA always produces optimal water loss and near-optimal salt gain solutions, on average, for any number of fireflies (i.e. the FA always generated near-optimal solution in each of the 30 runs). Conversely, at $t=$ 100 , the average water loss and salt gain values indicate that there can be some variability in the quality of the solution obtained irrespective of the number of fireflies employed in the FA process. However, the solutions are all very good. The indication from this examination is that the more iterations used, the better the solution quality obtained by the FA. 
Table 6. Average Salt Gain (\%) and Water Loss (\%) for Different Parameter Settings of the Firefly

Algorithm

\begin{tabular}{|l|l|l|l|l|l|l|l|l|l|l|l|l|}
\hline $\begin{array}{l}\text { No. of } \\
\text { Fireflies }\end{array}$ & \multicolumn{10}{|l|}{ Number of Iterations } \\
\hline & $\mathbf{1 0 0}$ & $\mathbf{2 5 0}$ & $\mathbf{5 0 0}$ & $\mathbf{1 0 0 0}$ & $\mathbf{1 5 0 0}$ & $\mathbf{2 5 0 0}$ \\
\hline & SG & WL & SG & WL & SG & WL & SG & WL & SG & WL & SG & WL \\
\hline & 3.00 & 45.01 & 2.69 & 42.60 & 2.89 & 43.24 & 2.93 & 43.67 & 2.88 & 45.03 & 2.93 & 45.03 \\
\hline $\mathbf{2 0}$ & 3.04 & 45.04 & 2.89 & 45.04 & 2.96 & 45.04 & 2.96 & 45.04 & 2.94 & 45.04 & 2.90 & 45.03 \\
\hline $\mathbf{5 0}$ & 2.94 & 45.01 & 2.95 & 45.03 & 3.02 & 45.04 & 3.03 & 45.04 & 2.95 & 45.04 & 2.98 & 45.04 \\
\hline $\mathbf{1 0 0}$ & 3.00 & 45.04 & 2.93 & 45.04 & 2.92 & 45.03 & 2.92 & 45.04 & 3.01 & 45.04 & 2.93 & 45.04 \\
\hline $\mathbf{1 5 0}$ & 3.00 & 45.04 & 2.92 & 45.04 & 2.92 & 45.04 & 2.94 & 45.04 & 3.01 & 45.03 & 2.98 & 45.04 \\
\hline $\mathbf{2 0 0}$ & 2.98 & 45.04 & 2.99 & 45.03 & 3.03 & 45.04 & 2.98 & 45.04 & 2.98 & 45.04 & 3.01 & 45.04 \\
\hline $\mathbf{2 5 0}$ & 3.04 & 45.03 & 2.94 & 45.04 & 2.99 & 45.03 & 2.93 & 45.04 & 2.99 & 45.04 & 3.03 & 45.04 \\
\hline $\mathbf{5 0 0}$ & & & & & & & & & & & & \\
\hline
\end{tabular}

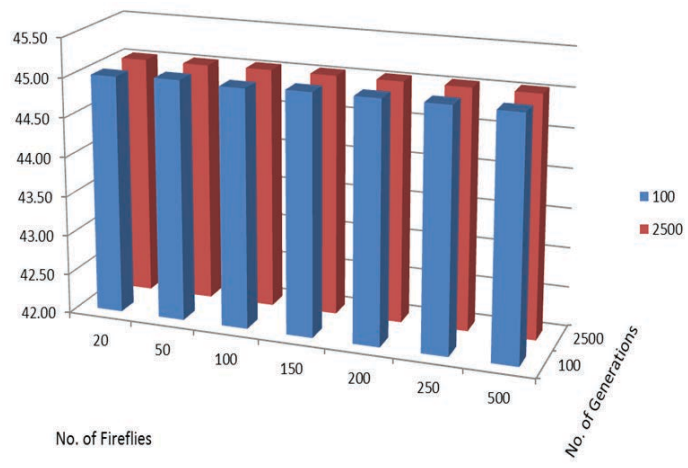

Figure 3. Comparison of Average Water Loss (\%) in the Mushrooms from Runs of 100 Generations and 2500 Generations in the Firefly Algorithm

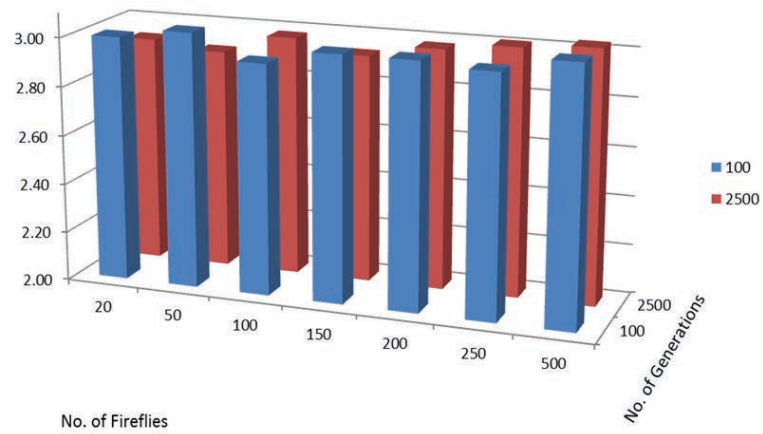

Figure 4. Comparison of Average Salt Gain (\%) in the Mushrooms from Runs of 100 Generations and

2500 Generations in the Firefly Algorithm

Similar to the preceding analysis, Figures 5 and 6 provide a comparison of the average water loss and salt gain responses obtained for the minimum and maximum number of fireflies considered. From these Figures, it can be seen that at $n=500$ fireflies, the FA always produces near-optimal water loss and salt gain solutions, on average, for any number of iterations. Namely, the average solutions are all extremely close to optimal. Conversely, at $n=20$ fireflies, the average water loss and salt gain values show that there can be considerable variability in the quality of the solution obtained irrespective of the number of iterations employed in the FA. These findings clearly illustrate that the more fireflies used in the FA, the better the solution quality.

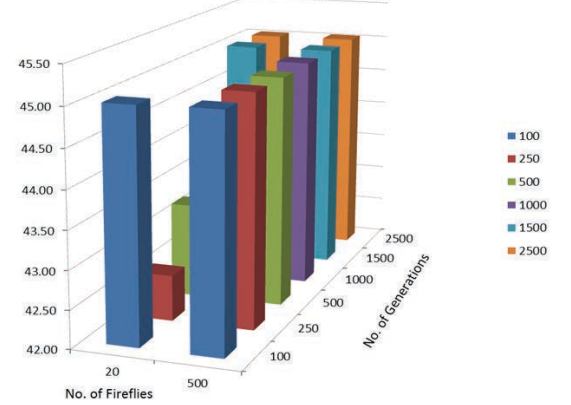

Figure 5. Comparison of Average Water Loss (\%) in the Mushrooms from Runs with 20 Fireflies and Runs with 500 Fireflies

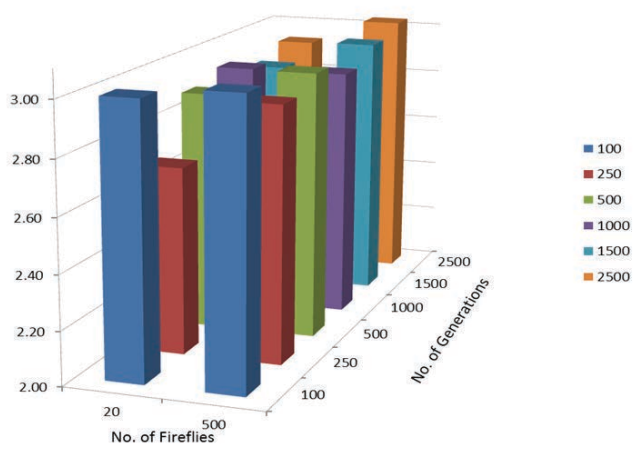

Figure 6. Comparison of Average Salt Gain (\%) in the Mushrooms Between Runs with 20 Fireflies and Runs with 500 Fireflies 
It should be further reinforced that Table 6 shows the average response values for each combination of $n$ and $t$. While the FA in the larger parameter value combinations tend to converge to the overall optimal solution, the smaller combinations would also frequently produce this optimal value within their set of the 30 runs. However, there would also be occasions where divergent solution values were found within the runs, thereby distorting the overall averages. Given the running time complexities for the FA, a combination of a relatively smaller value of $n$ combined with a relatively larger value for $t$ would be preferable from both a solution time and solution accuracy perspective. Table 6 shows that even the intermediate values in the experimental ranges considered for $n$ and $t$ tend to consistently produce very high quality solutions. The experimentation for this specific problem would indicate that the value for $t$ needs to be set somewhere in the range of 500 to 1000 iterations, while the value for $n$ should be between 100 and 150 fireflies if always being able to calculate the true optimal solution is required.

\section{Conclusion}

In this study, an empirical response surface approach was employed to provide the functional form of the osmotic dehydration responses for mushrooms. Using these estimates of the functional form, the resulting optimization model was formulated into a non-linear goal programming problem. The optimal solution to the goal programming problem was found using a computationally efficient, FA-directed procedure and the osmotic parameters determined were shown to be superior to those found in all previous instances. Computational experimentation on the goal programming model tested the relative solution effectiveness of the FA over its key running-time parameters of the number of iterations and the number of fireflies. This experimentation demonstrated that for intermediate-to-high values of either of the two key parameters, the FA would always determine overall optimal solutions, while lower values of either parameter produced greater variability in the solution quality. Since the running time complexity of the FA is linear in the number of iterations but polynomial in the number of fireflies, these results would seem to confirm that it would be more computationally practical to run the FA using a relatively larger number of iterations in combination with a "reasonable" number of fireflies than vice versa. Since an FA can clearly be modified to solve a diverse spectrum of "real world" problems beyond the context of fresh produce dehydration, the computational findings from this sensitivity analysis can obviously be extended into numerous other "real world" settings. These extensions will be considered in future research.

\section{References}

[1] Geohive World Crop Production www.geohive.com/charts/ag_crops.aspx 2014.

[2] B.K. Mehta, S.K. Jain, G.P. Sharma, V.D. Mugdal, R.C. Verma, A. Doshi and H.K. Jain, Optimization of Osmotic Drying Parameters for Button Mushroom (Agaricus bisporus), Applied Mathematics, 3(10A), 2012, 1298-1305.

[3] M.E. Venturini, J.E. Reyes, C.S. Rivera, R. Oria and D. Blanco, Microbiological Quality and Safety of Fresh Cultivated and Wild Mushrooms Commercialized in Spain, Food Microbiology, 28(8), 2011, 1492-1498.

[4] M.D. Rosa and F. Giroux, Osmotic treatments and problems related to the solution management, Journal of Food Engineering, 49(3), 2001, 223-236.

[5] S.K. Jain and R.C. Verma, Osmotic dehydration: A new, promising and emerging industry, Beverage and Food World, 30(1), 2003, 30-34.

[6] N.K. Rastogi, K.S.M.S. Raghavarao, K. Niranjan and D. Knorr, Recent developments in osmotic dehydration: Method to enhance mass transfer, Food Science Technology, 13(1), 2002, 48-59.

[7] J. Hawkes and J.M. Fink, Osmotic Concentration of Fruit Slices Prior to Dehydration, Food Processing Preservation, 2(4), 1978, 265-267.

[8] B.D. Shukla and S.P. Singh, Osmo-Convective Drying of Cauliflower, Mushroom and Green Pea, Food Engineering, 80(2), 2007, 741-747.

[9] R.V. Tonon, A.F. Baroni and M.D. Hubinges, Osmotic Dehydration of Tomato in Ternary Solutions: Influence of Process Variables on Mass Transfer Kinetics and an Evaluation of the Retention of Arytenoids, Food Engineering, 82(4), 2007, 509-517.

[10] A. Nieto, M.A. Castro and A. Alzamora, Kinetics of moisture transfer during air drying of blanched and/or osmotically dehydrated mango, Journal of Food Engineering, 50(2), 2001, 175-185. 
[11] S.K. Jain, R.C. Verma, L.K. Murdia, H.K. Jain and G.P. Sharma, Optimization of Process Parameters for Osmotic Dehydration of Papaya Cubes, Food Science and Technology, 48(2), 2011, 211-217.

[12] A. Kar and D.K. Gupta, Osmotic dehydration characteristics of button mushrooms, Journal of Food Science and Technology, 38(4), 2001, 352-357.

[13] N.S. Sodhi, N. Singh and K. Komal, Osmotic dehydration kinetics of carrots, Journal of Food Science and Technology, 43(4), 2006, 374-376.

[14] D. Torreggiani and G. Bertolo, Osmotic pretreatments in fruit processing: chemical, physical and structural effects, Journal of Food Engineering, 49(30), 2001, 247-253.

[15] R. Imanirad and J.S. Yeomans, Fireflies in the Fruits and Vegetables: Combining the Firefly Algorithm with Goal Programming for Setting Optimal Osmotic Dehydration Parameters of Produce, in Recent Advances in Swarm Intelligence and Evolutionary Computation. X-S. Yang (ed.), Springer, Heidelberg, Germany, 2015, 49-69.

[16] J.S. Yeomans, Computing Optimal Food Drying Parameters Using the Firefly Algorithm, Journal on Computing, 4(1), 2014a, 40-44.

[17] J.S. Yeomans, Establishing Optimal Dehydration Process Parameters for Papaya By Employing A Firefly Algorithm, Goal Programming Approach, International Journal of Engineering Research and Applications, 4(9), 2014b, 145-149.

[18] J.S. Yeomans and X.S. Yang, Determining Optimal Osmotic Drying Parameters Using the Firefly Algorithm, International Conference on Applied Operational Research (ICAOR), Vancouver, Canada, July 29-31, 2014a.

[19] G.E. Box and D.W. Behnken, Some New Three Level Designs for the Study of Quantitative Three Variables, Technometrics, 2(4), 1960, 455-475.

[20] R.H. Myers and D.C. Montgomery, Response Surface Methodology: Process and Product Optimization Using Designed Experiments, John Wiley and Sons, New York, USA, 1995.

[21] D.C. Montgomery, Design and Analysis of Experiments $4^{\text {th }}$ Ed., John Wiley and Sons, New York, USA, 1997.
[22] R. Imanirad, X.S. Yang, and J.S. Yeomans, Modelling-to-Generate-Alternatives Via the Firefly Algorithm, Journal of Applied Operational Research, 5(1), 2013, 14-21.

[23] X.S. Yang, Nature-Inspired Metaheuristic Algorithms $2^{\text {nd }}$ Ed., Luniver Press, Frome, UK, 2010.

[24] J.S. Yeomans and X.S. Yang, Municipal Waste Management Optimization Using A Firefly Algorithm-Driven Simulation-Optimization Approach, International Journal of Process Management and Benchmarking, 4(4), 2014b, 363-375.

[25] J.S. Yeomans and R. Imanirad, A Parametric Testing of the Firefly Algorithm in the Determination of the Optimal Osmotic Drying Parameters for Papaya, IEEE Symposium Series on Computational Intelligence (IEEE SSCI) Special Session on Theory and Applications of Nature-Inspired Optimization Algorithms, Orlando, USA, December 9-12, 2014.

[26] M.S. Alam, A. Singh and B.K. Sawhney, Response Surface Optimization of Osmotic Dehydration Process for Aonla Slices, Food Science Technology, 47(1), 2010, 47-54.

[27] G.S. Mudhar, R.T. Toledo, J.D. Floros and J.J. Jen, Optimization of carrot dehydration process using response surface methodology, Journal of Food Science, 54(11), 1989, 714-719.

[28] L. Shi, C.H. Xue, Y. Zhao, Z.J. Li, X.Y. Wang and D.L. Luan, Optimization of Processing Parameters of Horse Mackerel (Trachurus Japonicus) Dried in a Heat Pump Dehumidifier Using Response Surface Methodology, Food Engineering, 87(1), 2008, 74-81.

[29] M.B. Uddin, P. Amsworth and S. Ibanoglu, Evaluation of Mass Exchange during Osmotic Dehydration of Carrots Using Response Surface Methodology, Food Engineering, 65(4), 2004, 473-477.

[30] S. Ranganna, Handbook of analysis and quality control for fruits and vegetable products, Tata McGraw Hill Publishing, New Delhi, India, 1986.

[31] J.S. Yeomans, Simulation-Driven Optimization in Waste Management Facility Expansion Planning, Journal of Computational Methods in Sciences and Engineering, 12(1/2), 2012, 111-127. 